\title{
The correlation between pharyngeal residue, penetration/aspiration and nutritional modality: a cross-sectional study in patients with neurogenic dysphagia
}

\author{
Correlazione tra residui faringei, penetrazione/aspirazione e modalità nutrizionali: \\ uno studio cross-sectional in pazienti con disfagia neurogena
}

\author{
Sara Nordio, Arianna Di Stadio, Isabella Koch, Paola Stritoni, Francesca Meneghello, Katie Palmer \\ Fondazione Ospedale San Camillo IRCCS, Venice, Italy
}

\begin{abstract}
SUMMARY
Aspiration risk has a substantial influence on clinical management of swallowing disorders, and can be associated with pharyngeal residue. The aims of this cross-sectional study are to examine the correlation between the presence of pharyngeal residue and penetrationaspiration during fiberoptic endoscopic evaluation of swallowing (FEES), examine the correlation between objective data and Functional Oral Intake Scale (FOIS) and determine whether using objective assessment (Pooling score and Penetration Aspiration Scale-PAS) to categorise patients as pathological or not identifies the same patients identified by FOIS. Fifty-five patients with neurogenic dysphagia were evaluated during FEES by using the Pooling Score scale and PAS. They underwent an assessment of nutritional modalities using FOIS. There was a significant positive correlation between Pooling score and PAS scores for semisolid bolus (Pearson $=0.305 ; \mathrm{p}=0.024$ ) and liquids (Pearson $=0.841 ; \mathrm{p}=0.000$ ). The semi-solid bolus Pooling score had a negative correlation with FOIS (Pearson $=-0.355$; $\mathrm{p}=0.008$ ), but there were no other significant correlations for FOIS with Pooling score or PAS. There were significant differences between objective assessments (P-score/PAS) and functional measure (FOIS) for identifying patients as pathological; although the positive predictive values were high, the negative predictive values were very low. Although pharyngeal residues are significantly associated with the presence of penetration-aspiration during endoscopy, the real intake modalities are not correlated with objective assessments of swallowing disorders. Therefore, clinicians need to implement a comprehensive approach to assess dysphagia.
\end{abstract}

KEY WORDS: deglutition disorders, residues, penetration, aspiration

\section{RIASSUNTO}

Il rischio di aspirazione ha una sostanziale influenza nella gestione dei disturbi della deglutizione, e può essere associato a residui faringei. Gli scopi di questo studio cross-sectional sono di indagare la correlazione tra la presenza di residui faringei e penetrazionelaspirazione durante la fiberoptic endoscopic evaluation of swallowing (FEES), indagare la correlazione tra i dati oggettivi e la Functional Oral Intake Scale (FOIS); verificare se la valutazione oggettiva (Pooling score e Penetration Aspiration Scale-PAS) per dividere i pazienti tra patologici e non patologici, identifica gli stessi pazienti identificati dalla FOIS. 55 pazienti con disfagia neurogena sono stati valutati durante la FEES utilizzando la Pooling Score e la PAS, poi sono state valutate le modalità nutrizionali utilizzando la FOIS. C'è una significativa correlazione positiva tra i punteggi della Pooling Score e della PAS per i boli semisolidi (Pearson =0,305; $p=0,024)$ e liquidi (Pearson $=0,841 ; p=0,000)$. I punteggi della Pooling score riguardanti i boli semi-solidi mostrano una correlazione negativa con la FOIS (Pearson =- 0,355; $p=0,008$ ), ma non ci sono altre correlazioni significative per la FOIS confrontata con la Pooling score o la PAS. Ci sono differenze significative tra le valutazioni oggettive (P-score e PAS) e la misurazione funzionale (FOIS) per identificare i pazienti come patologici; sebbene $i$ valori predittivi positivi siano alti, $i$ valori predittivi
Received: March 2, 2018

Accepted: June 4, 2018

Published on line: March 25, 2019

\section{Correspondence}

Sara Nordio

Fondazione Ospedale San Camillo IRCCS, via Alberoni 70, 30126 Venice, Italy

Tel. +39041 2207189. Fax +39041731330

E-mail: sara.nordio@ospedalesancamillo.net

\section{Funding}

None.

Conflict of interest

The Authors declare no conflict of interest.

How to cite this article: Nordio S, Di Stadio A, Koch I, et al. The correlation between pharyngeal residue, penetration/aspiration and nutritional modality: a cross-sectional study in patients with neurogenic dysphagia. Acta Otorhinolaryngol Ital 2020;40:38-43. https:// doi.org/10.14639/0392-100X-2136

(C) Società Italiana di Otorinolaringoiatria e Chirurgia Cervico-Facciale

\section{(c) (1) $(9)$}

This is an open access article distributed in accordance with the CC-BY-NC-ND (Creative Commons Attribution-NonCommercial-NoDerivatives 4.0 International) license. The article can be used by giving appropriate credit and mentioning the license, but only for non-commercial purposes and only in the original version. For further information: https:// creativecommons.org/licenses/by-nc-nd/4.0/deed.en 
negativi sono molto bassi. Nonostante i residui faringei siano significativamente associati alla presenza di penetrazione-aspirazione durante l'esame endoscopico, le reali modalità di nutrizione non sono correlate con le valutazioni oggettive dei disturbi di deglutizione. Quindi, $i$ clinici necessitano di implementare un approccio globale per valutare la disfagia.

PAROLE CHIAVE: disturbi deglutitori, residui, penetrazione, aspirazione

\section{Introduction}

Oropharyngeal dysphagia is a symptom that negatively affects swallowing dynamics, involving the oral, pharyngeal, or oesophageal phases ${ }^{1}$. Oropharyngeal dysphagia is commonly associated with neurologic disease, as well as other acute and chronic conditions. Prevalence rates of oropharyngeal dysphagia are $37 \%$ and $78 \%$ in the acute and chronic phases of stroke, respectively ${ }^{2}$. Patients affected by neurodegenerative diseases have high prevalence of this symptom; in fact, $82 \%$ of patients with Parkinson's Disease ${ }^{3}$ and $25 \%$ of patients with amyotrophic lateral sclerosis ${ }^{4}$ are symptomatic for oropharyngeal dysphagia. The presence of dysphagia is associated with increased risk of pulmonary complications, increased hospital length of stay, dehydration, malnutrition and mortality ${ }^{5}$.

Aspiration risk is one of the primary factors that influences the clinical management of swallowing disorders ${ }^{6}$. We define "aspiration" as the passage of material below the level of the vocal folds, while penetration is defined as the passage of material into the larynx that does not pass below the vocal folds ${ }^{7}$. From a clinical point of view, the amount of the material and the depth of the aspiration acquired is also highly relevant, influencing the rehabilitation process and clinical outcomes. Patients who aspirate food and liquids into the airway are at increased risk of developing pneumonia ${ }^{8}$. Factors associated with increased aspiration risk include salivary pooling ${ }^{9}$, impaired sensation ${ }^{10}$, reduced airway protection ${ }^{11}$ and pharyngeal residue ${ }^{12,13}$. Murray et al. reported a significant association between accumulated secretions and aspiration of food and/or drink, observed during endoscopic evaluation of older individuals, including patients with mixed aetiology and healthy persons ${ }^{9}$.

Pharyngeal residue suggests an underlying impairment of oropharyngeal bolus driving forces ${ }^{14}$ and reduced swallow efficiency ${ }^{15}$. In neurogenic dysphagia, residue consists in the result of incomplete bolus clearance caused by poor propulsion, weak pharyngeal muscles activity and/or impaired upper oesophageal sphincter relaxation ${ }^{12,16}$. Pharyngeal bolus residue is most commonly located in the valleculae and/or the pyriform sinuses ${ }^{17}$.

Clinical judgment and some previous studies ${ }^{18-20}$ suggest that pharyngeal residue can influence aspiration risk, as it can be seen during instrumental swallowing examinations ${ }^{6}$. Videofluoroscopic swallowing study and fibreoptic endoscopic evaluation of swallowing (FEES) are consid- ered the gold standards for detecting dysphagia and aspiration ${ }^{21}$ and both provide images of post-swallow pharyngeal residue. They can be considered complementary, and both are needed to make a correct diagnosis of dysphagia. However, a recent study by Kelly et al. ${ }^{6}$ suggests that pharyngeal residue and aspiration are better identified by FEES rather than videofluoroscopy. This superior sensitivity is most likely related to the axial, direct view of the surface anatomy within the laryngopharynx ${ }^{22}$. The only protocol for assessing the presence and amount of pharyngeal residues that has been validated in Italy is the Pooling Score scale by Farneti et al. ${ }^{23,24}$.

The first aim of this study is to verify a correlation between the presence and amount of pharyngeal residues and penetration-aspiration measured during FEES, assessed with the Pooling Score ${ }^{23,24}$ and Penetration Aspiration Scale(PAS) ${ }^{7}$. The second aim is to verify the correlation and relationship between the degree of severity of dysphagia considering objective data as pharyngeal residue and penetration/aspiration and Functional Oral Intake Scale (FOIS) ${ }^{25}$. In addition, as a third aim, we examine if currently available assessments (Pooling Score, PS, FOIS) have the same specificity and sensitivity for identifying dysphagic patients.

\section{Materials and methods}

We analysed in a prospective study 55 patients with neurogenic dysphagia hospitalised at San Camillo Hospital IRCCS, Venice, Italy from February 2017 until August 2017. The study was approved by the Ethics Committee of IRCCS San Camillo Foundation, and research was conducted in accordance to the Declaration of Helsinki. All participants signed informed consent before being included in the study. Patients were referred for a FEES after a bedside clinical swallowing examination performed by an expert speech and language pathologist reporting dysphagia. The FEES was performed by a trained otorhinolaryngologist, assisted by a speech and language pathologist, using a Storz endoscope. No topical anaesthetic was used; a watersoluble lubricant was used to minimise patient discomfort. First, 5-mL yogurt for pureed ${ }^{26}$ food was used, followed by $5-\mathrm{mL}$ water for liquid food. They were mixed with one drop of blue dye in each milliliter, to improve visualisation during endoscopy and avoid confounding food with salivary 
secretion. We did not test solids because of the discomfort to patients after the other two trials. All fluids were given at fridge temperature to minimise the risk of aspiration. We avoided testing liquids in patients who had a compromised ability to swallow their own saliva and aspiration during pureed to minimise the possibility of aspiration during FEES. In this case $(n=7)$, the lowest score for each assessment scale was given to patients who could not assume liquid bolus during the evaluation. The entire clinical procedure was recorded on video, and the videotape of the procedure was analysed by an otolaryngologist (P.S.) and a speech and language pathologist (I.K.). For each bolus ingested, a pooling score according to Farneti's Scale ${ }^{23}$ was given, where pooling of materials is considered as any material in the containment cavities of the hypopharynx and larynx before and/ or after the act of swallowing ${ }^{23}$. Two different parameters were assessed. First, the pooling-score considers the location (identified by anatomical landmarks), amount of pooling materials and management (ability of the patient to clear the residue). Second, data from Pooling-Sensation Collaboration Age score (P-SCA) combines this information with additional data, such as sensation of the pharynx, patient collaboration and age. Both are continuous variables, with a minimum score corresponding to no dysphagia, and a high score to severe dysphagia. Scores range from 4-11 for the Pooling-score and 3-16 for the Pooling-SCA score.

Penetration-aspiration status was evaluated using the eightpoint PAS Scale ${ }^{7}$, where a score of 1 indicates no airway invasion and a score of 8 indicates silent aspiration below the level of the vocal cords.

The speech and language therapist completed the FOIS, which is an ordinal scale where level 1 corresponds to "nothing by mouth" and 7 is a "total oral diet without restrictions".

Demographic data (age and sex), clinical presentation (aetiology, presence of tracheostomy, tube feeding, presence of cognitive deficits) were documented by a neurologist. Data are shown in Table I.

For data analysis, we used two-tailed Pearson correlation to examine the correlation between the presence and magnitude of pharyngeal residues (Pooling score and PoolingSCA) and penetration-aspiration of materials in the airways (PAS), for both semisolid and liquid bolus. Second, Pearson correlations were calculated to identify the correlations between the level of dysphagia expressed by presence and amount of residues (Pooling score) or penetration-aspiration (PAS) and the clinical assessment of dysphagia based on nutritional modalities (FOIS). We choose to not consider the P-SCA score in statistical analysis due to inclusion of clinical characteristics in the scale. Third, to investigate whether using the objective scales during FEES (Pooling score and
Table I. Patient characteristics.

\begin{tabular}{|c|c|c|}
\hline & Mean & $\mathrm{SD}^{*}$ \\
\hline Age (year) & 62.6 & 14.6 \\
\hline Time since onset (months) & 41 & 116.5 \\
\hline Aetiology & \multicolumn{2}{|c|}{ Frequency } \\
\hline $\begin{array}{l}\text { Ischaemic stroke } \\
\text { Cerebellar stroke } \\
\text { Haemorrhagic stroke } \\
\text { Subarachnoid haemorrhage } \\
\text { Traumatic brain Injury } \\
\text { Amyotrophic lateral sclerosis } \\
\text { Wallemberg syndrome } \\
\text { Parkinson's disease } \\
\text { Multiple sclerosis } \\
\text { Anoxia } \\
\text { Arnold Chiari malformation } \\
\text { Bleeding after arteriovenous malformation rupture } \\
\text { Spinal cord injury } \\
\text { Cerebellar ataxia }\end{array}$ & & \\
\hline Tracheal tube & \multicolumn{2}{|c|}{39} \\
\hline Tube feeding & \multicolumn{2}{|c|}{44} \\
\hline Cognitive impairment & \multicolumn{2}{|c|}{45} \\
\hline
\end{tabular}

PAS) to dichotomously categorise patients as dysphagic or non-dysphagic, could identify the same patients as the nutritional modalities assessment (FOIS). Pooling-score scores of less than six were considered not pathological, and scores of six or more as pathological. For PAS, scores of two or more were considered pathological. A score of less than 7 was considered pathological for FOIS. Chi square was used to assess whether there was a difference in the categorisation of patients as pathological or not by Pooling-score or PAS compared to FOIS. We also report the positive and negative predictive values and specificity and sensitivity values. Significance was set at $\mathrm{p}<0.05$. All statistical analyses were conducted using IBM SPSS Statistics version 20.

\section{Results}

We recruited 55 patients, 41 males and 14 females. Patient age ranged from 20 to 84 (mean age $=62.6$ years; $\mathrm{SD}=14.6)$, the mean disease onset at time of evaluation was $41.0(\mathrm{SD}=116.5)$ and the most common diagnosis was haemorrhagic stroke (Tab. I). 39 had tracheal tube and 44 tube feeding; 45 had cognitive impairment (Tab. I). Mean scores on the different assessment scales are reported in Table II.

Correlations between the presence and amount of pharyngeal residues measured by Pooling Score ${ }^{23}$ and the penetration-aspiration of materials in the airway assessed with PAS ${ }^{7}$ are shown in Table III. 
Table II. Mean scores on the battery of tests.

\begin{tabular}{lcccc} 
& Minimum & Maximum & Mean & SD $^{3}$ \\
Pooling-score semisolids & 2 & 8 & 6.0 & 1.6 \\
Pooling-SCA semisolids & 3 & 15 & 6.6 & 2.5 \\
PAS' semisolids & 1 & 8 & 2.3 & 2.0 \\
Pooling-score fluids & 4 & 11 & 7.6 & 2.3 \\
Pooling SCA fluids & 3 & 16 & 3.1 & 4.2 \\
PAS' fluids & 1 & 8 & 3.5 & 2.4 \\
FOIS $^{2}$ & 0 & 7 & & 1.7 \\
\hline
\end{tabular}

${ }^{1}$ : penetration aspiration scale; ${ }^{2}$ : functional oral intake scale; ${ }^{3}$ : standard deviation.

There was a significant positive correlation between Pooling-score and PAS scores for both semisolid and liquid bolus. Significant positive correlations were also seen for Pooling-SCA and PAS scores, for both semisolid and liquid bolus.

The correlation between Pooling score or PAS with FOIS is reported in Table IV. All correlations were in a negative direction but none were significant, except that Pooling-score for semisolid bolus was negatively correlated with FOIS $(\mathrm{p}=0.008)$.

The third aim was to examine whether using the scores from objective evaluation (Pooling score and PAS) to dichotomously categorise patients as pathological or not identified the same patients as the functional/symptomatic scale

Table III. Correlation between pooling score and penetration aspiration scale.

\begin{tabular}{|c|c|c|}
\hline & \multicolumn{2}{|c|}{$\begin{array}{l}\text { Penetration aspiration scale } \\
\text { (PAS) }\end{array}$} \\
\hline & Pearson & $P$ value \\
\hline Semisolids pooling-score & 0.305 & $0.024^{*}$ \\
\hline Semisolids pooling-SCA ${ }^{1}$ & 0.373 & $0.005^{*}$ \\
\hline Liquids pooling-score & 0.841 & $0.000^{*}$ \\
\hline Liquids pooling-SCA ${ }^{1}$ & 0.852 & $0.000^{*}$ \\
\hline
\end{tabular}

: sensibility, collaboration, age; : $: p<0.05$.

Table IV. Correlation between pooling score/PAS and the functional oral intake scale.

\begin{tabular}{|c|c|c|}
\hline & \multicolumn{2}{|c|}{ FOIS $^{2}$} \\
\hline & Pearson & $P$ value \\
\hline Semisolids pooling-score & -0.355 & $0.008^{*}$ \\
\hline Semisolids pooling-SCA ${ }^{3}$ & -0.134 & 0.330 \\
\hline Semisolids PAS ${ }^{1}$ & -0.201 & 0.140 \\
\hline Liquids pooling-score & -0.180 & 0.189 \\
\hline Liquids pooling-SCA ${ }^{3}$ & -0.122 & 0.375 \\
\hline Liquids PAS ${ }^{1}$ & -0.218 & 0.110 \\
\hline
\end{tabular}

: : penetration aspiration scale; ${ }^{2}$ : functional oral intake scale; ${ }^{3}:$ sensibility, collaboration, age; $: p<0.05$.
(FOIS). There were no significant differences between the instruments (P-score/PAS) and clinical functional measure (FOIS) in identifying patients as pathological. We separately evaluated the specificity and sensitivity of the assessment for liquid and semisolid. FOIS, compared with PAS for identifying dysphagia for liquids, had a sensitivity of $6.3 \%$ and a specificity of $94.9 \%$. For semisolids, the sensitivity was $6.1 \%$ and specificity was $95.5 \%$. The sensitivity of FOIS, when compared with P-score for identifying dysphagia in swallowing liquids, was $10 \%$ and specificity was $97.1 \%$. For semisolids the sensitivity and specificity were $13.6 \%$ and $100 \%$, respectively. Negative and positive predictive values are shown in Table V.

\section{Discussion}

Our study identified three main findings. First, Poolingscore, Pooling-SCA and PAS scores are positively correlated for both semisolid and liquid bolus. Second, there were no significant correlations between Pooling-score/PAS and FOIS, except for semisolid pooling score compared with FOIS. There were differences between the objective assessments (Pooling-score/PAS) and the functional measure (FOIS) in identifying pathological dysphagia; in fact, although the sensitivity of Pooling/PAS is high, the specificity is lower than FOIS.

The correlation between the objective assessment shows that the presence, position and quantity of pharyngeal residue is related to an increased risk of penetration and/or aspiration in neurogenic dysphagia. These results are in accordance with the data by other authors 9,12,27.

The accumulation of residue in neurological is due to a reduction in tongue thrust strength, impaired pharyngeal constriction, or failure to release of upper oesophageal sphincter. Furthermore, the incomplete elevation of hyo-laryngeal complex, impaired motility of vocal cords and incomplete epyglottidis tilting increase the risk of penetration-aspiration events.

The deficit function of trigeminal (III), glossopharyngeal (IX), vagus (X) and hypoglossal (XII) nerves modifies the 
Table V. Positive predictive values for pathological categorisation of the pooling-score and penetration-aspiration scale (PAS) compared to the functional oral intake scale (FOIS).

\begin{tabular}{lccc} 
& Negative predictive value for FOIS & Positive predictive value for FolS $^{2}$ & \\
Semi-solid pooling-score & 13.6 & 100.0 & 0.590 \\
Semi-solid penetration-aspiration scale & 6.1 & 95.5 & 0.807 \\
Liquid pooling-score & 10.0 & 97.1 & 0.546 \\
Liquid penetration-aspiration scale & 6.3 & 94.9 & 0.869 \\
\hline
\end{tabular}

${ }^{1}$ : calculated with chi square; ${ }^{2}$ : functional oral intake scale.

swallowing process by acting at different levels, both in the oropharyngeal and in the oesophageal phases. Specifically, the association between residues and penetration-aspiration can be explained by considering the main role of cranial nerve $\mathrm{X}$ and the consequences of its damage, because it allows pharyngeal propulsion and cough reflex. Thus, it is relevant to evaluate its function to predict the increased risk in patients with neurogenic dysphagia.

In our results, all correlations between Pooling score or PAS with FOIS were in a negative direction and only Pooling-score values for semisolid bolus was negatively correlated with FOIS. This latter scale documents the functional level of oral intake food and liquid, also considering the use of enteral nutrition.

In neurological patients, enteral nutrition may be necessary to avoid malnutrition in cases of severe dysphagia ${ }^{28}$.

Our results showed that there was no correlation between FOIS and scores that describe pooling and penetrationaspiration level, for both semisolids and fluids. Therefore, objective evaluation describes fundamental aspects of dysphagia, but, especially in neurological patients, clinical outcome can be influenced by other important parameters, for example, awareness, collaboration, cognitive abilities, presence of apraxia, or speech impairment like aphasia, which can compromise the ability to understand language. The presence of these types of impairments in neurological disorders ${ }^{29-32}$ can add further difficulties in the deglutition process, even more if specific or complex postures and manoeuvres are requested to swallow safely, and may reduce the success rate of swallowing treatment. It is important to highlight that more than $80 \%$ of our sample had cognitive impairment, which may explain the discrepancies observed in the results of penetration-aspiration assessment and FOIS.

Swallowing is a complex behaviour that requires dynamic neural coordination at both the cerebral and brainstem levels ${ }^{31}$. The primary sensory and motor cortex, supplementary motor area, prefrontal and inferior frontal cortex, cingulate cortex, insula, basal ganglia, thalamus and cerebellum ${ }^{32}$ (cortical and sub-cortical structures) need to have perfect functionality to produce an effective swallow- ing process. The analysis of the comparison between dichotomised pathological values is consistent with the other results of this study; in fact, the sensitivity was low for all the comparisons made, and therefore only a small number of patients non-pathological on the P-score or PAS scale were equally non-pathological according to FOIS.

Overall, our results support the idea that the three scales used provide diverse information about different aspects of dysphagia, and thus all three are necessary to perform accurate diagnosis and adapt the rehabilitation path to the specific dysphagia characteristics of each patient by designing a sort of "personal therapy".

There are several limitations in this study. First, the sample size was small and there was a heterogeneity in the patient aetiologies. We made the swallowing test with one bolus only of semisolid and one of fluid. We did not have access to information about whether residue remains over time. Finally, in the third aim of the study, we used specific cut-offs to dichotomise the scores into pathological or not pathological categories. The results of the study may differ if different cut-offs were used, and this would be an interesting avenue for future research to adjust the cut-offs to find an optimum way of assessing the objective and clinical aspects.

Future research would be necessary to evaluate possible differences in the aspect of dysphagia in specific neurological population (stroke, traumatic brain injury etc.), to evaluate if by swallowing several boluses there is an improvement or a worsening of dysphagia and to study if the position of residue may be relevant for evaluating the increased risk of penetration-aspiration over time.

\section{Conclusions}

In conclusion, in this study we have shown that pharyngeal residues, measured through Farneti's protocol, are significantly associated with the presence of penetrationaspiration on swallowing semisolid and liquid bolus during endoscopic evaluation in neurological patients, reported by PAS. Moreover, the tool that documents the functional impact of dysphagia of oral intake of food and liquid (FOIS) 
is not correlated to the objective assessment of dysphagia. Therefore, each scale measures different significant aspects and the use of all three assessment is necessary for an accurate diagnosis of the disorder. In our opinion, it is necessary to implement a comprehensive approach to assess neurogenic dysphagia, including evaluation of presence and amount of pharyngeal residues, penetration-aspiration and effective nutritional modalities, in order to plan the proper treatment and monitor changes in swallowing efficacy and impact on functional feeding abilities.

\section{References}

1 World Health Organization. International classification of disease (ICD); 2014. www.who.int/classifications/icd/en

2 Martino R, Foley N, Bhogal S, et al. Dysphagia after stroke: incidence, diagnosis, and pulmonary complications. Stroke 2009;36:2756-63. https://doi.org/10.1161/01.STR.0000190056.76543.eb

3 Kalf JG, de Swart BJM, Bloem BR, et al. Prevalence of oropharyngeal dysphagia in Parkinson's disease: a meta-analysis. Parkinsonism Relat Disord 2012;18:311-5. https://doi.org/10.1016/j.parkreldis.2011.11.006

4 Trail M, Nelson N, van JN, et al. Major stressors facing patients with amyotrophic lateral sclerosis (ALS): a survey to identify their concerns and to compare with those of their caregivers. Amyotroph Lateral Scler Other Motor Neuron Disord 2004;5:40-5. https://doi. org/10.1080/14660820310016075

5 Wilson RD. Mortality and cost of pneumonia after stroke for different risk groups. J Stroke Cerebrovasc Dis 2012;21:61-7. https://doi. org/10.1016/j.jstrokecerebrovasdis.2010.05.002

6 Kelly AM, Leslie P, Beale T, et al. Fibreoptic endoscopic evaluation of swallowing and videofluoroscopy: does examination type influence perception of pharyngeal residue severity? Clin Otolaryngol 2006;31:425-32. https://doi.org/10.1111/j.1749-4486.2006.01292.x

7 Rosenbek JC, Robbins JA, Roecker EB, et al. A penetration-aspiration scale. Dysphagia 1996;11:93-8.

8 Pikus L, Levine MS, Yang YX, et al. Videofluoroscopic studies of swallowing dysfunction and the relative risk of pneumonia. Am J Roentgenol 2003;180:1613-6. https://doi.org/10.2214/ajr.180.6.1801613

9 Murray J, Langmore SE, Ginsberg S, et al. The significance of accumulated oropharyngeal secretions and swallowing frequency in predicting aspiration. Dysphagia 1996;11:99-103.

10 Aviv JE, Kim T, Sacco RL, et al. FEESST: a new bedside endoscopic test of the motor and sensory components of swallowing. Ann Otol Rhinol Laryngol 1998;107:378-87. https://doi. org/10.1177/000348949810700503

11 Lundy DS, Smith C, Colangelo L, et al. Aspiration: cause and implications. Otolaryngol Head Neck Surg 1999;120:474-8. https://doi. org/10.1053/hn.1999.v120.a91765

12 Eisenhuber E, Schima W, Schober E, et al. Videofluoroscopic assessment of patients with dysphagia: pharyngeal retention is a predictive factor for aspiration. AJR Am J Roentgenol 2002;178:393-8. https:// doi.org/10.2214/ajr.178.2.1780393

13 Oliveira DL, Moreira EAM, de Freitas MB, et al. Pharyngeal residue and aspiration and the relationship with clinical/nutritional status of patients with oropharyngeal dysphagia submitted to videofluoroscopy. J Nutr Health Aging 2017;21:336-41. https://doi.org/10.1007/ s12603-016-0754-6

14 Dejaeger E, Pelemans W, Ponette E, et al. Mechanisms involved in postdeglutition retention in the elderly. Dysphagia 1997;12:63-7. https://doi.org/10.1007/PL00009520

15 Rademaker AW, Pauloski BR, Logemann JA, et al. Oropharyngeal swallow efficiency as a representative measure of swallowing function. J Speech Hear Res 1994;37:314-25. https://doi.org/10.1044/ jshr.3702.314

16 Omari TI, Dejaeger E, Tack J, et al. An impedance-manometry based method for non-radiological detection of pharyngeal postswallow residue. Neurogastroenterol Motil 2012;24:e277-84. https://doi. org/10.1111/j.1365-2982.2012.01931.x

17 Logemann JA. Evaluation and treatment of swallowing disorders. Austin, Tex: PRO-ED; 1998.

18 Horner J, Buoyer FG, Alberts MJ, et al. Dysphagia following brain-stem stroke. Clinical correlates and outcome. Arch Neurol 1991;48:1170-3. https://doi.org/10.1001/archneur.1991.00530230078026

19 Perlman AL, Grayhack JP, Booth BM. The relationship of vallecular residue to oral involvement, reduced hyoid elevation, and epiglottic function. J Speech Hear Res 1992;35:734-41. https://doi.org/10.1044/ jshr.3504.734

20 Han TR, Paik N, Park JW. Quantifying swallowing function after stroke: a functional dysphagia scale based on videofluoroscopic studies. Arch Phys Med Rehabil 2001;82:677-82. https://doi.org/10.1053/ apmr.2001.21939

21 Bours GJJW, Speyer R, Lemmens J, et al. Bedside screening tests vs. videofluoroscopy or fibreoptic endoscopic evaluation of swallowing to detect dysphagia in patients with neurological disorders: systematic review. J Adv Nurs 2009;65:477-93. https://doi.org/10.1111/j.13652648.2008.04915.x

22 Kaneoka AS, Langmore SE, Krisciunas GP, et al. The boston residue and clearance scale: preliminary reliability and validity testing. Folia Phoniatr Logop 2013;65:312-7. https://doi.org/10.1159/000365006

23 Farneti D. Pooling score: an endoscopic model for evaluating severity of dysphagia. Acta Otorhinolaryngol Ital 2008;28:135-40.

24 Farneti D. The Pooling-score (P-score): inter- and intra- rater reliability in endoscopic assessment of the severity of dysphagia. Acta Otorhinolaryngol Ital 2014;34:105-10.

25 Crary MA, Carnaby-Mann GD, Groher ME. Initial psycometric assessment of a functional oral intake scale for dysphagia in stroke patients. Arch Phys Med Rehabil 2005;86:1516-20. https://doi. org/10.1016/j.apmr.2004.11.049.

26 Chichero JAY, Lam P, Steele CM. Development of international terminology and definitions for texture-modified food and thickened fluids used in dysphagia management: the IDDSI Framework. Dysphagia 2017;32:293-314. https://doi.org/10.1007/s00455-016-9758-y

27 Molfenter SM, Steele CM. The relationship between residue and aspiration on the subsequent swallow: an application of the normalized residue ratio scale. Dysphagia 2013;28:494-500. https://doi. org/10.1007/s00455-013-9459-8

28 Ojo O. Enteral feeding tubes: not perfect but necessary. Br J Nurs 2015;24:910. https://doi.org/10.12968/bjon.2015.24.18.910

29 Meijer KA, Eijlers AJC, Douw L, et al. Increased connectivity of hub networks and cognitive impairment in multiple sclerosis. Neurology 2017;88:2107-14. https://doi.org/10.1212/WNL.0000000000003982

30 Hanagasi HA, Tufekcioglu Z, Emre M. Dementia in Parkinson's disease. J Neurol Sci 2017;374:26-31. https://doi.org/10.1016/j. jns.2017.01.012

31 Hochstenbach J, Mulder T, van Limbeek J, et al. Cognitive decline following stroke. J Clin Exp Neuropsychol 1998;20:503-17. https:// doi.org/10.1076/jcen.20.4.503.1471

32 Azouvi P, Arnould A, Dromer E, et al. Neuropsycology of traumatic brain injury: an expert overview. Nev Neurol 2017;173:461-72. https://doi.org/10.1016/j.neurol.2017.07.006 